\title{
Development of optimization method for plate heat exchanger with undulation
}

\author{
Václav Dvořák ${ }^{1, a}$ \\ ${ }^{1}$ Technical university of Liberec, Faculty of mechanical engineering, Department of Power Engineering Equipment. Address: \\ Studentska 2, 46117, Liberec, Czech Republic
}

\begin{abstract}
The article deals with optimization of undulated heat transfer surface of plate heat exchanger. The goal of optimization is not only to increase effectiveness of heat transfer but also to reduce the pressure drop. A combined pattern of undulation which combines herringbone pattern and wavy pattern was optimized and best values of four parameters were found; angle of herringbone pattern, number, phase and amplitude of longitudinal waves of wavy pattern. The optimization procedure looked for maximum of objective function which was a linear combination of effectiveness and pressure drop. We used simple Monte Carlo method and the optimum was searched for four values of reference pressure drop. Four different optimization were run and we investigated the effect of various definition of objective function and parameters of undulation. It was found that during optimization of combined pattern, the herringbone pattern is more favoured than wavy pattern. It is caused by the fact that herringbone pattern was described by the only one free parameter, which was the angle of undulation, and therefore it is more likely to be found by a stochastic method. This assumption was confirmed when simple wavy pattern was optimized and higher values of objective function and effectiveness were found.
\end{abstract}

\section{Introduction}

Energy recovery during air ventilation is one of the ways to reduce the energy consumption of buildings. Heat recovery can substantially reduce energy consumption needful for heating during cold seasons or air conditioning during hot seasons. The main component of any energy recovery devices are heat exchangers. Development of recuperative heat exchangers in recent years has focused on increasing their effectiveness. Another challenge is the development of so-called enthalpy exchangers for simultaneous heat and moisture transport, i.e. transport of both sensible and latent heat, as presented by Vít et al. in work [1]. To develop heat exchangers, methods of computational fluid dynamics are increasingly used, but there are still some problems to transfer the calculation results to the design and manufacturing processes.

To simulate a heat exchanger, it is necessary to create a model and a computational mesh and then use computational fluid dynamic (CFD) software. When assembling the heat exchanger, complicated and irregular narrow channels are created. These channels are split into small volumes (elements). The final meshes are structured or unstructured with different element sizes. Most of researchers use unstructured meshes for their calculations.
Gherasim et al., in work [2], presented a comparison of various grids for a plate heat exchanger modelled by a tetrahedral mesh. In order to assess the influence of the grid resolution on the solution, five grids were created and tested by meshing the volumes with different interval sizes. Laminar and turbulent regimes were simulated. It was discovered that the two grids with the smallest elements provided very similar results. In terms of temperature, the obtained results were close for grids with smaller elements. For pressure, quite a large difference between the grid with smallest elements and the grid with the largest ones was discovered.

Subsequent researchers have used numerical simulations to investigate plate heat exchangers with chevron (undulated) profiles, as it is presented in Figure 1. Tsai [3] and Liu [4], for example, dealt with these heat exchangers and their different geometries. Their conclusions regarding temperature and pressure loss were similar to Gherasim [2].

Similarly Giurgiu et al. in work [5] used CFD to study the influence of geometric characteristics of the two plates on the intensification process of heat transfer. They used SST k- $\omega$ turbulence model and non-structural highdensity mesh and modelled only small part of the heat exchanger to analyse mini channels with the inclination angles of $30^{\circ}$ respectively $60^{\circ}$. They carried out also a session of experimental measurements which confirms the results obtained through numerical simulation that the

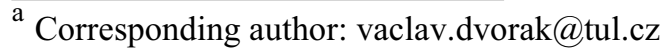


plate heat exchanger model using mini channels with inclination angle of $\beta=60^{\circ}$ provides best heat transfer. Kumar et al. [6] experimentally investigated the effect of symmetric and mixed chevron angles in a plate heat exchanger on heat transfer rate ratio, friction losses and many others quantities. Experimental observation confirmed previously known optimum for enhancement in heat transfer.

Rios Iribe et al. [7] investigated, using CFD and hexahedral meshes, momentum and heat transfer of a non-Newtonian fluid in steady laminar regime through a plate heat exchanger at constant wall temperature. They investigated the effect of number of plates and distance between plates on the heat transfer and the pressure drop and developed empirical correlations. Although the flow pattern was highly complex, under an adequate definition of the characteristics parameters, it was possible to establish simple correlations between the dimensionless numbers that characterize the thermal-hydraulic behavior of plate heat exchangers.

Al-Waked et al. [8] investigated numerically conjugate heat and mass transfer processes across membrane heat exchangers of variable mass transfer resistance. The authors studied effects of channel height, Reynolds number and flow direction on heat exchanger thermal effectiveness and energy recovered. A hybrid mesh was used.

Novosád and Dvořák [9], investigated the influence of oblique waves in herringbone pattern on the heat transfer surface. The main difficulty faced in this work was the creation of custom geometry. Each version had to be modelled, meshed, loaded into the solver, set the boundary conditions and subsequently perform the numerical simulation separately.
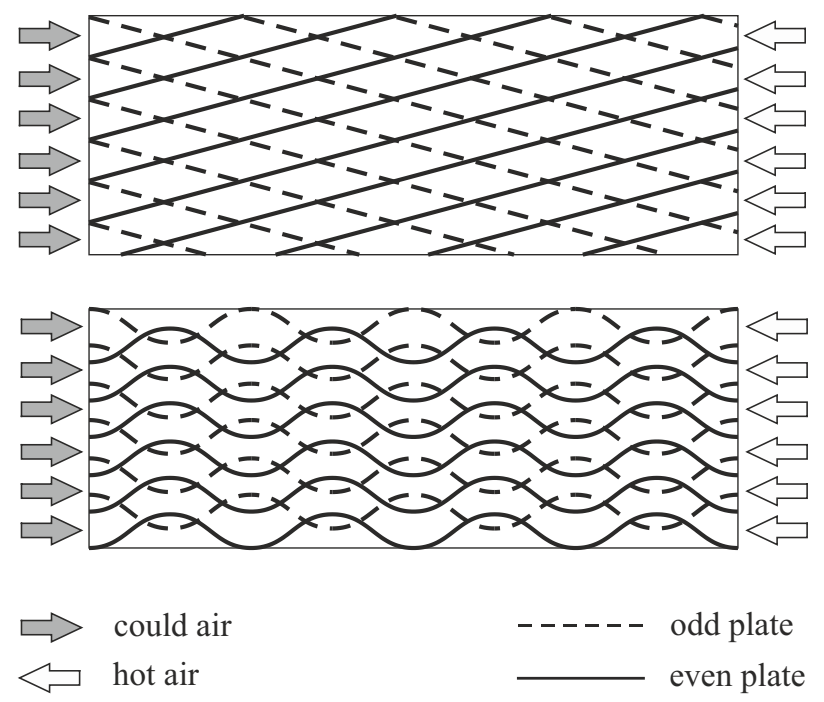

Figure 1 Two basic types of undulation; up - herringbone pattern, down - wavy pattern with sinusoidal waves.

Waves in herringbone pattern are widely used for steal plate heat exchanger. The reason is that forming the plates provides efficient and reliable way to increase the heat transfer area of the heat exchanger. Another pattern is the wavy pattern, visible down in Figure 1, which provides the same advantages. Although it is often used in plastic and aluminium plate heat exchangers in air ventilation systems, it is overlooked by researchers and it is not clear, which of both patterns is better, i.e. has higher heat transfer and lower pressure drop.

Therefore the aim of this work is to develop a method and optimize the combined pattern to answer these questions.

\section{Methods}

\subsection{Methods of grid generation}

To optimize a plate heat exchanger, we needed to model, mesh and compute a lot of computational variants. However, there are several disadvantages of repeated generation of computational meshes: Demanding and time consuming work and parameterization of the model is problematic. Further, even a small change of geometry requires to do again the whole process of model creation and mesh generation. Furthermore, meshes are not similar, i.e. the size, shape, and height of cells adjacent to walls are not the same for different topologies and numerical error which is added to the solution and influence evaluation of objective function.

Therefore, we used method, developed by Dvořák [10], for generating computational variants. This method is based on a dynamic mesh, which is provided by Ansys Fluent software. The meshes were created by pull procedure, which is similar to the own production process, i.e. the pulling direction is perpendicular to the plates. The main advantage is that this type of mesh generation is automatic and controlled by in-house software. All computational variants thus have the same mesh.

In this paper, we discuss the case of a counter flow heat exchanger, which does not have a symmetrical heat transfer area. It is because undulation cross each other, as it is obvious form Figure 1. To simulate flow and heat transfer in such heat exchanger, we need to create model containing two plates, as it is shown in Figure 2.

The heat transfer surface was divided into two parts. Input and output portions with length of $L_{0}=0.05 \mathrm{~m}$ were fixed, remained flat and served for developing velocity profiles before and after central part.

The central part which was provided by undulation had length $L=0.15 \mathrm{~m}$. Input boundary conditions were specified by velocities of $3 \mathrm{~m} / \mathrm{s}$, the output boundary conditions were specified as pressure outlets with static pressure $0 \mathrm{~Pa}$. Bottom and upper boundary conditions were periodic, thus a heat exchanger with infinite number of plates was simulated. The width of the model was $B=0.05 \mathrm{~m}$. We used mesh density, element size and count of cell layers according to recommendations from Dvořák and Novosád [11].

In this study, we used turbulence model SST k- $\omega$, medium was air considered as incompressible gas. As a results, we obtained pressure, velocity, turbulence and temperature fields inside the computational domain for given inlet velocity. 


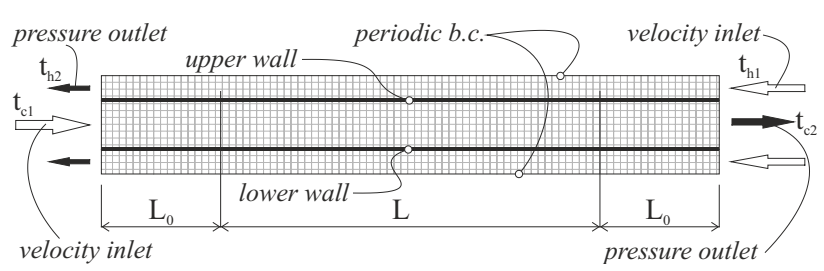

Figure 2 Model of heat exchange surface of counter flow recuperative heat exchanger.

In this work, the undulation was created as combination of two patterns, as it is indicated in Figure 3. Firstly, it was undulations in herringbone pattern, see top of Figure 1, defined by angle of undulation $\alpha$ (rad). Secondly, it was undulation in wavy patter, see bottom of Figure 1, defined by number of longitudinal waves $n(1)$, their amplitude $a(\mathrm{~m})$ and phase $f(1)$.

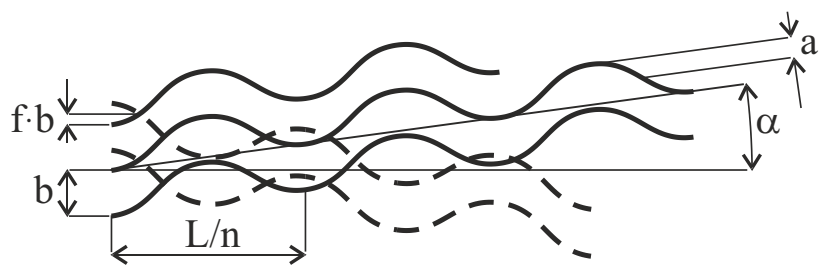

Figure 3 Parameters of plate undulation for optimization.

The shape of the middle part of plates was defined as vertical displacement $z(\mathrm{~m})$ of points on the wall according to equation

$$
z=\frac{p}{2}[\cos (2 \pi m)+s]
$$

where $p=0.003 \mathrm{~m}$ is plate pitch, $s=1$ for upper wall of the model and $s=-1$ for lower wall. $m$ (1) represents wave number, which is calculated from horizontal coordinates $(x, y)$ of points on the wall and set of parameters according to relation

$$
m=\frac{f}{2}+\frac{1}{b}\left[s y+\frac{a}{2} \cos \left(2 \pi \frac{n x}{L}\right)+y^{\prime}\right],
$$

where $f(1)$ is phase, which is important especially for wavy corrugation, $b=0.006 \mathrm{~m}$ is pitch of corrugation, see Figure 3. $a(\mathrm{~m})$ is amplitude and $n(1)$ count of longitudinal waves according to the same figure, while $L(\mathrm{~m})$ is the length of the middle part of the model heat exchanger. These parameters are needful for wavy corrugation.

For angled corrugation in herringbone patter, parameter $y^{\prime}$ is implied as

$$
y^{\prime}=-x \tan \alpha .
$$

Amplitude of wavy corrugation was determined by two ways. First, it was defined as multiplication of pitch of corrugation according to relation

$$
a=\zeta_{1} b,
$$

but this way can make it difficult to find optimal $\zeta_{1}$ together with optimal count of longitudinal waves $n$. Therefore we also tried relation

$$
a=\zeta_{2} \frac{L}{n},
$$

in which the amplitude depends on parameter $\zeta_{2}$ and the length of longitudinal waves. The method of grid transformation according to relations (1) to (5) are described in work [10].

\subsection{Method for optimization of plate heat exchanger}

Two optimize a heat exchanger, we need an objective function $F$. Producers of heat exchangers often require as high heat transfer as it is possible for given pressure drop. Therefore and according to previous works [12], we used linear combination of two main characteristic properties of a heat exchanger in the form of

$$
F=\eta-C_{F}\left(\frac{\left|\Delta p-\Delta p_{R}\right|}{\Delta p_{R}}\right)^{R}
$$

where $\Delta p(\mathrm{~Pa})$ is calculated or measured pressure drop, $\Delta p_{R}(\mathrm{~Pa})$ is reference or required pressure loss, $\eta(1)$ is calculated or measured coefficient of effectiveness, $C_{F}(1)$ penalty coefficient and $R(1)$ is parameter by which we can control peak of objective function. Thus, obtained effectiveness is penalized by the difference between obtained and required pressure drop.

The pressure drop was defined as pressure difference

$$
\Delta p=\bar{p}_{01}-\bar{p}_{2},
$$

where $\bar{p}_{01}(\mathrm{~Pa})$ is mass-averaged total pressure in the inlet and $\bar{p}_{2}(\mathrm{~Pa})$ is area-averaged static pressure at the pressure outlet.

The coefficient of effectiveness (or effectiveness) is the efficiency of sensible heat transfer. Most recuperative heat exchangers in air conditioning systems work in isobaric mode, where mass flow rates of hot and cold air are equal, i.e. $\dot{m}_{h}=\dot{m}_{c}$. Assuming parity between specific heat capacities $c_{p h}=c_{p c}$, we can write the effectiveness as

$$
\eta=\frac{t_{h 1}-t_{h 2}}{t_{h 1}-t_{c 1}}
$$

where $t_{h 1}\left({ }^{\circ} \mathrm{C}\right)$ and $t_{h 2} \quad\left({ }^{\circ} \mathrm{C}\right)$ are inlet and outlet temperatures of hot air, $t_{c 1}\left({ }^{\circ} \mathrm{C}\right)$ is outlet temperature of cold air.

The task of optimization dealt with finding of optimal values of four parameters according to Figure 3; angle of undulation $\alpha$, number of longitudinal waves $n$, their amplitude $a$ and phase $f$, while plate pitch $p$, often defined by requirement on material consumption, and pitch of undulation $b$, which is usually defined by 
deformability of plate material and technology of pressing, remained constant during optimization.

As optimization method, we used a simple Monte Carlo algorithm. To emphasize searching for local optimum, we used modification that favoured variants closer to the last best variant found according to relations

$$
\begin{gathered}
\pi_{i}^{n}=\pi_{i}^{\text {best }}-\operatorname{ran}^{2}\left(\pi_{i}^{\text {best }}-\pi_{i}^{\text {low }}\right), \\
\pi_{i}^{n}=\pi_{i}^{\text {best }}+\operatorname{ran}^{2}\left(\pi_{i}^{u p}-\pi_{i}^{\text {best }}\right),
\end{gathered}
$$

where $\pi_{i}^{n}$ is a new value of optimization parameter $\pi_{i}$, $\pi_{i}^{\text {best }}$ is the value of the parameter for the last best variant, $\pi_{i}^{\text {low }}$ and $\pi_{i}^{u p}$ are lower and upper limit of the same parameter respectively, and $\operatorname{ran}=\langle 0 ; 1\rangle$ is random number. The use of equations (9) and (10) were alternated also randomly.

\section{Results}

The model of plate heat exchanger was optimized for four values of reference pressure, $\Delta p_{R}=100,150,200$ and $250 \mathrm{~Pa}$. The optimization was initialized by values of parameters: $\alpha=5^{\circ}, n=4, \zeta_{1}=0.5$, which corresponds to $\zeta_{2}=0.08$, and $f=0.25$.

There are a typical course of first 100 steps of optimization in Figure 4, where pressure drop, effectiveness and objective function are presented for reference pressure drop $\Delta p_{R}=100 \mathrm{~Pa}$ and parameters of equation (6) $R=1$ and $C_{F}=0.5$. We can see how the objective function is reduced while pressure drop varies significantly from reference pressure.

Figure 5 shows development of parameters of best variants for the same optimization. We can observe, how the phase $f$ and angle $\alpha$ become negligible while the amplitude of longitudinal waves represented by ratio $a / b=\zeta_{1}$ increased. It means that the wavy undulation was found as optimal in this case.

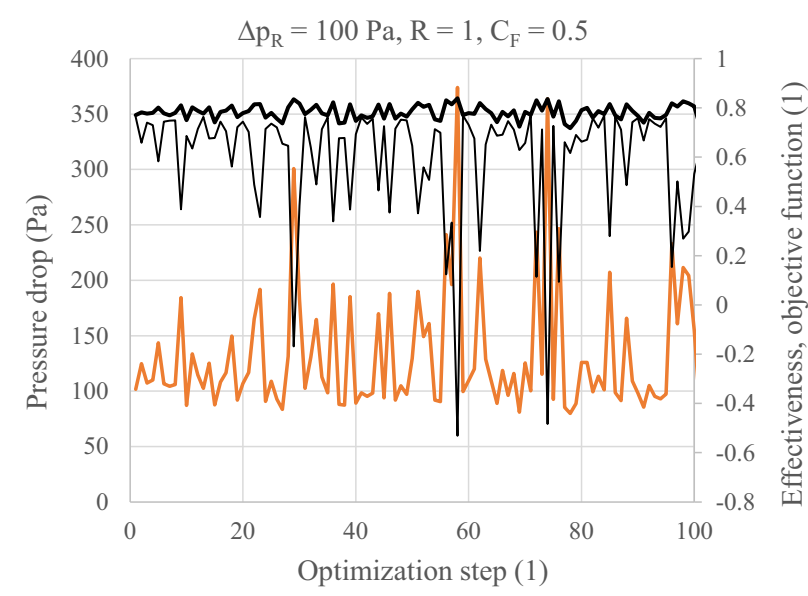

— Pressure drop —Effectiveness — Objective function

Figure 4 Course of optimization for reference pressure $\Delta p_{R}=100 \mathrm{~Pa}, R=1$ and $C_{F}=0.5$.
Resulsts of optimization for different refference pressure are in Table 1 . We can see that for refference pressure 150 and $200(\mathrm{~Pa})$, the angle is high, while parameter $a / b$ is low. It means that herringbone pattern was found as optimal in these cases. The results for refference pressure $250(\mathrm{~Pa})$ show combination of both patterns.

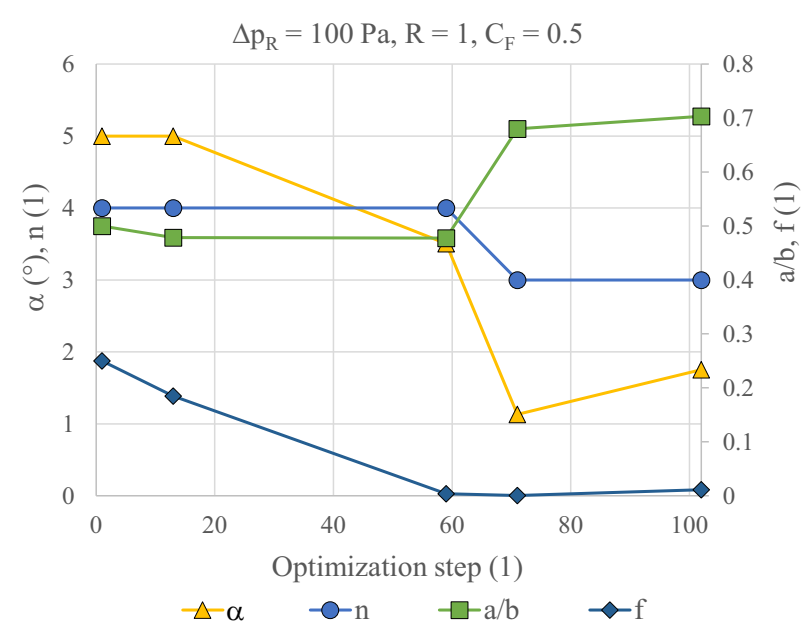

Figure 5 Development of parameters of best variants during optimization for reference pressure $\Delta p_{R}=100 \mathrm{~Pa}, R=1$ and $C_{F}=0.5$.

Table 1. Results of optimization no. 1 for various values of reference pressure $\Delta p_{R}, R=1$ and $C_{F}=0.5$.

\begin{tabular}{|l|c|c|c|c|}
\hline$\Delta p_{R}(\mathrm{~Pa})$ & 100 & 150 & 200 & 250 \\
\hline No. of steps & 102 & 93 & 109 & 17 \\
\hline$\eta(1)$ & 0.7719 & 0.8117 & 0.8263 & 0.8309 \\
\hline$\Delta p(\mathrm{~Pa})$ & 100.2 & 150.0 & 200.0 & 251.1 \\
\hline$F(1)$ & 0.7709 & 0.8116 & 0.8263 & 0.8288 \\
\hline$\alpha\left(^{\circ}\right)$ & 1.8 & 22.7 & 28.8 & 29.9 \\
\hline$n(1)$ & 3 & 3 & 2 & 5 \\
\hline$a / b(1)$ & 0.70 & 0.12 & 0.10 & 0.49 \\
\hline$f(1)$ & 0.01 & 0.07 & 0.29 & 0.30 \\
\hline
\end{tabular}

Because the peak of the objective function is quite steep and narrow, the parameters of equation (6) were changed in the second run of optimization. We used $R=2$ and $C_{F}=5$. Results of the second run of optimization are given in Table 2. Around five hundred variation were calculated, but the last best variants were found much earlier.

We can see from Table 2 that parameter $a / b$ was reduced almost to zero for three lower values of required pressure. Thus undulation in herringbone pattern was found as optimal in these cases with angle $\alpha$ increasing with reference pressure. Waves were necessary only in case of the highest reference pressure $250 \mathrm{~Pa}$. In that case, angle of undulation almost reached its maximum, $30^{\circ}$, and waves were necessary to increase obtained pressure drop. We can also see that obtained values of objective function were higher for the second run of 
optimization. It indicates that parameters $R=2$ and $C_{F}=5$ better suite the optimization procedure.

Table 2. Results of optimization no. 2 for various values of reference pressure $\Delta p_{R}, R=2$ and $C_{F}=5$.

\begin{tabular}{|l|r|r|r|r|}
\hline$\Delta p_{R}(\mathrm{~Pa})$ & 100 & 150 & 200 & 250 \\
\hline No. of steps & 126 & 161 & 76 & 90 \\
\hline$\eta(1)$ & 0.7789 & 0.8123 & 0.8268 & 0.8317 \\
\hline$\Delta p(\mathrm{~Pa})$ & 101.0 & 151.2 & 201.1 & 251.5 \\
\hline$F(1)$ & 0.7784 & 0.8120 & 0.8266 & 0.8315 \\
\hline$\alpha\left(^{\circ}\right)$ & 10.5 & 23.1 & 28.9 & 29.5 \\
\hline$n(1)$ & 7 & 3 & 6 & 7 \\
\hline$a / b(1)$ & 0.01 & 0.02 & 0.00 & 0.36 \\
\hline$f(1)$ & 0.34 & 0.18 & 0.26 & 0.37 \\
\hline
\end{tabular}

If we compare results of Table 1 and Table 2, we find differences in values of obtained parameters. For the second run of optimization, herringbone pattern was found, while for the first run, it seems that combination of pattern was preferred. Therefore, the third run of optimization worked with different definition of amplitude $a$ of longitudinal waves, and parameter $\zeta_{2}$ defined by relation (5) was used instead of $\zeta_{1}$.

Table 3. Results of optimization no. 3 for various values of reference pressure $\Delta p_{R}, R=2, C_{F}=5$ and $\zeta_{2}=0.08$ as initial value.

\begin{tabular}{|l|c|c|c|c|}
\hline$\Delta p_{R}(\mathrm{~Pa})$ & 100 & 150 & 200 & 250 \\
\hline No. of steps & 176 & 117 & 76 & 74 \\
\hline$\eta(1)$ & 0.7794 & 0.8120 & 0.8268 & 0.8381 \\
\hline$\Delta p(\mathrm{~Pa})$ & 101.1 & 150.9 & 201.1 & 256.4 \\
\hline$F(1)$ & 0.7787 & 0.8118 & 0.8266 & 0.8349 \\
\hline$\alpha\left(^{\circ}\right)$ & 10.8 & 23.0 & 28.9 & 1.6 \\
\hline$n(1)$ & 5 & 5 & 6 & 8 \\
\hline$n \cdot a / L(1)$ & 0.00 & 0.00 & 0.00 & 0.27 \\
\hline$f(1)$ & 0.48 & 0.47 & 0.26 & 0.17 \\
\hline
\end{tabular}

We can see from Table 3 that results of the third run of optimization were almost the same as for the second run. The only one difference are results for the highest reference pressure for which wave pattern with negligible angle $\alpha=1.6^{\circ}$ was found in the third run of optimization. Nevertheless, this exception lead to higher objective function and because it was still unclear which pattern is more advantage a fourth optimization was run with angle $\alpha=0$ to optimize only the wave pattern.

Results of optimization of wavy pattern are collected in Table 4. Firstly, we can see that the best variants were changing even after more than 250 steps. Such slow optimization is probably connected with complexity of wavy pattern which is described by three parameters. So it seems that during optimization of combined pattern, undulation in herringbone patter is more favoured and more likely to be the result of optimization. Secondly, values of obtained effectiveness and objective function are higher than values obtained while undulation in herringbone patter was found.

Table 4. Results of optimization of wavy pattern for various values of reference pressure $\Delta p_{R}, R=2, C_{F}=5, \alpha=0$, $\zeta_{2}=0.08$ as initial value.

\begin{tabular}{|l|c|c|c|c|}
\hline$\Delta p_{R}(\mathrm{~Pa})$ & 100 & 150 & 200 & 250 \\
\hline No. of steps & 236 & 273 & 269 & 279 \\
\hline$\eta(1)$ & 0.7796 & 0.8135 & 0.8282 & 0.8402 \\
\hline$\Delta p(\mathrm{~Pa})$ & 100.0 & 153.3 & 202.5 & 248.8 \\
\hline$F(1)$ & 0.7796 & 0.8112 & 0.8274 & 0.8401 \\
\hline$\alpha\left(^{\circ}\right)$ & \multicolumn{5}{|c}{0} \\
\hline$n(1)$ & 5 & 4 & 5 & 5 \\
\hline$n \cdot a / L(1)$ & 0.05 & 0.18 & 0.23 & 0.26 \\
\hline$f(1)$ & 0.24 & 0.20 & 0.18 & 0.25 \\
\hline
\end{tabular}

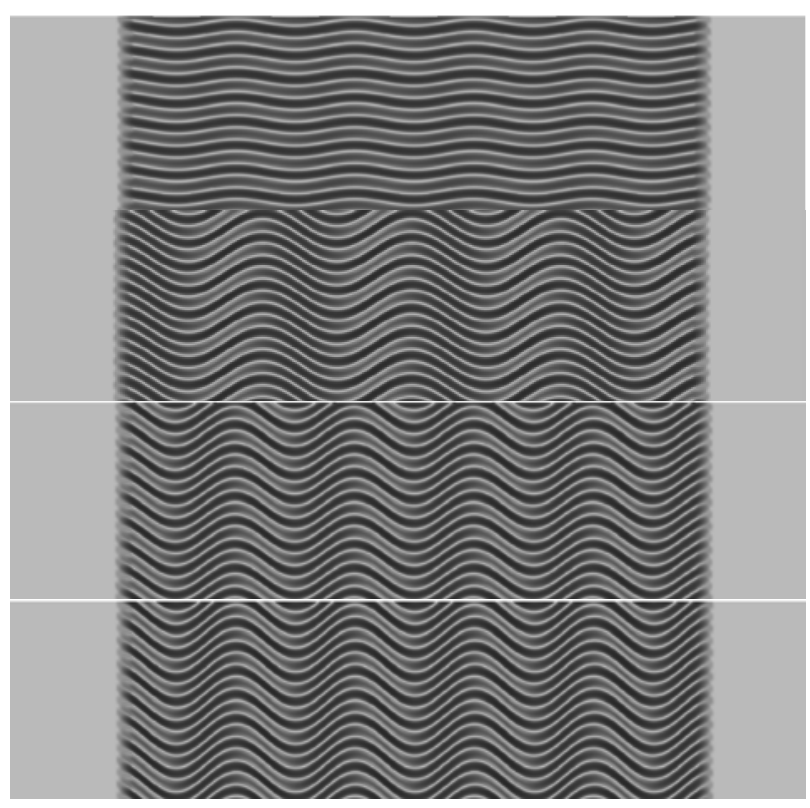

Figure 6 Optimized shapes of heat transfer surfaces for reference pressures $100,150,200$ and $250 \mathrm{~Pa}$.

\section{Conclusions}

An undulated heat transfer surface of recuperative heat exchanger was optimized to obtain as high coefficient of effectiveness of heat transfer as possible. We optimized a combined pattern of undulation which combines herringbone pattern and wavy pattern. The task of optimization was to find optimal values of four parameters; angle of herringbone pattern, number, phase and amplitude of longitudinal waves of wavy pattern.

The optimization procedure looked for maximum of objective function which was a linear combination of effectiveness and pressure drop. We used simple Monte Carlo method and the optimum was searched for four values of reference pressure drop. Four different optimization were run and we investigated the effect of various definition of objective function and parameters of undulation. 
It was found that during optimization of combined pattern, the herringbone pattern is more favoured than wavy pattern. It is probably caused by the fact that herringbone pattern was described by the only one free parameter, which was angle of undulation, and therefore it is more likely to be found by a stochastic method. This assumption was confirmed when simple wavy pattern was optimized and higher values of objective function and effectiveness were found. Nevertheless, optimization of wave pattern, which was defined by three free parameters, took longer time.

In further work, it would be beneficial to combine Monte Carlo method with a local optimization method to find optimums more precisely.

\section{Acknowledgment}

This publication was written at the Technical University of Liberec, Faculty of Mechanical Engineering with the support of the Institutional Endowment for the Long Term Conceptual Development of Research Institutes, as provided by the Ministry of Education, Youth and Sports of the Czech Republic in the year 2016.

\section{References}

1. T. Vít, P. Novotný, V.V. Nguyen, V. Dvořák, WSEAS, Recent Advances in Energy, Environment, Economics and Technological Innovation, 34-38 (2013)

2. I. Gherasim, N. Galanis, C.T. Nguyen, Int. Jour. of Thermal Science. 50, 1499-1511 (2011)

3. Y.Ch. Tsai, F.B. Liu, P.T. Shen, Int. Commun. in Heat and Mass Transfer 36, 574-578 (2009)

4. F.B. Liu, Y.Ch. Tsai, Heat Mass Transf. 46, 585-593 (2010)

5. O. Giurgiu, A. Pleşa, L. Socaciu. Energy Procedia 85, 244-251 (2016)

6. V. Kumar, A.K. Tiwari, S.K. Ghosh. Energy Conversion and Manag. 118, 142-154 (2016)

7. E.Y. Rios-Iribe, M.E. Cervantes-Gaxiola, E. RubioCastro, O.M. Hernández-Calderón, Applied Thermal Eng. (in press)

8. R. AL-Waked, M.S. Nasif, G. Morrison, M. Behnia, Applied Thermal Eng. 84, 301-309 (2015)

9. J. Novosád, V. Dvořák, EPJ Web of Conferences 67, 02085 (2014)

10. V. Dvořák, Recent Advances in Computer Science 1, 193-198 (2014)

11. V. Dvořák, J. Novosád, Recent Advances in Computer Science, pp 115-120 (2015)

12. V. Dvořák, Applied Mechanics \& Materials 752-753, 820-827 (2015). 